\title{
Modelos empíricos para estimar o acúmulo de matéria seca de capim-marandu com variáveis agrometeorológicas
}

\author{
Pedro Gomes da Cruz ${ }^{(1)}$, Patricia Menezes Santos(1), José Ricardo Macedo Pezzopane ${ }^{(1)}$, \\ Patrícia Perondi Anchão Oliveira ${ }^{(1)}$ e Leandro Coelho de Araujo(2)
}

\begin{abstract}
(1)Embrapa Pecuária Sudeste, Rodovia Washington Luiz, Km 234, CEP 13560-970 São Carlos, SP. E-mail: pgdcruz@gmail.com, patricia@cppse.embrapa.br, jricardo@cppse.embrapa.br, ppaolive@cppse.embrapa.br (2)Universidade de São Paulo, Escola Superior de Agricultura Luis de Queiroz, Avenida Pádua Dias, no 11, CEP 13418-900 Piracicaba, SP .E-mail: Ic_araujo@yahoo.com.br
\end{abstract}

Resumo - O objetivo deste estudo foi testar modelos empíricos de regressão linear, para a predição do acúmulo de matéria seca (TAMS) de Urochloa brizantha cv. Marandu, em função de variáveis agrometeorológicas. Para gerar os modelos, foi utilizada a taxa média de acúmulo de matéria seca, em condições de sequeiro, entre 1998 e 2002. As variáveis avaliadas foram: temperaturas mínima, máxima e média, radiação global (Rg), graus-dia, evapotranspiração real (ETR) e potencial (ETP) obtidas a partir do balanço hídrico, unidades fototérmicas (UF) e índice climático de crescimento (ICC). As regressões univariada e multivariada mostraram boa capacidade de predição, com exceção para as que utilizam a UF. Os melhores resultados foram para a regressão multivariada, com $T_{\text {min }}$, Rg e ETR: $R^{2}$, 0,84; raiz do quadrado médio do resíduo (RQMR), 14,72; e critério de informação de Akaike (CIA), 222,5. Na regressão linear univariada, destacaram-se as variáveis: graus-dia corrigido ( $\mathrm{R}^{2}, 0,75$; RQMR,17,84; e CIA, 242,6), temperatura mínima corrigida $\left(\mathrm{R}^{2}, 0,75\right.$; RQMR, 17,82; CIA, 244,1), e ICC ( $\mathrm{R}^{2}, 0,74$; RQMR, 17,85; CIA, 236,9). A correção das variáveis agrometeorológicas pela relação entre evapotranspiração real e potencial (ETR/ETP), em geral, melhora a predição da TAMS pelos modelos.

Termos para indexação: Brachiaria brizantha, Urochloa brizantha, regressão linear, regressão multivariada.

\section{Empirical models to estimate the accumulation of dry matter in Marandu palisade grass using agrometeorological variables}

\begin{abstract}
The objective of this work was to test empirical linear regression models, to predict dry matter accumulation rates (DMAR) of Urochloa brizantha $\mathrm{cv}$. Marandu, using agrometeorological variables. To generate the models, the average dry matter accumulation under rainfed conditions, between 1998 and 2002, was used. The evaluated variables were: minimum, maximum and average temperatures, global radiation (GR), degree-days, actual (AET) and potential evapotranspiration (PET) obtained from the water balance, photothermal units (PU) and the climatic growth index (CGI). Except for the PU, the univariate and multivariate regressions showed good predictive ability. The best results were for the multivariate regression, with $\mathrm{T}_{\text {min }}$, GR and AET: $\mathrm{R}^{2}, 0.84$; root mean square residual (RMSR), 14.72; and Akaike's information criterium (AIC), 222.5. In the univariate regression, the following variables stood out: corrected degree-days ( $\mathrm{R}^{2}, 0.75$; RMSR, 17.84; CIA, 242.6), corrected minimum temperature ( $\mathrm{R}^{2}$, 0.75; RMSR, 17.82; AIC, 244.1); and CGI ( $\mathrm{R}^{2}, 0.74$; RMSR, 17.85; AIC, 236.9). The correction of the agrometeorological variables using the relation between real and potential evapotranspiration (AET/PET) enhances, in general, the model prediction of DMAR.
\end{abstract}

Index terms: Brachiaria brizantha, Urochloa brizantha cv. Marandu, linear regressions, multivariate regressions.

\section{Introdução}

O Brasil apresenta características climáticas que favorecem a exploração de forrageiras tropicais com elevado potencial de produção de biomassa. Nos ecossistemas de pastagens, o potencial de produção pode ser determinado geneticamente; entretanto, fatores ambientais como temperatura, luminosidade e disponibilidade hídrica influenciam diretamente as respostas fisiológicas das pastagens (Zhu et al., 2008).
Nos últimos anos, a preocupação com o clima tornou-se um fato recorrente, principalmente com relação ao planejamento das atividades agrícolas. Os relatórios do Intergovernmental Panel on Climate Change (IPCC) indicam que perdas significativas na agricultura ocorrerão, caso as perspectivas de mudanças climáticas venham a se concretizar. Essas mudanças compreendem desde as variações consideradas naturais do regime climático até aquelas aceleradas por ações antrópicas (Solomon et al., 2007). 
Modelos de crescimento mecanísticos têm sido utilizados, recentemente, para simular o crescimento de pastagens tropicais e subtropicais (Rymph et al., 2004; Corson et al., 2007). Tais modelos são complexos e necessitam de conhecimentos sobre diversos processos fisiológicos da planta e sobre como cada variável ambiental influencia esses processos (Boote et al., 2002; Pedreira et al., 2011). Assim, a aplicação desses modelos em grande escala é difícil, pela falta de conhecimento dos processos fisiológicos que ocorrem no dossel, pela dificuldade de desenvolvimento de modelos que integrem todos os processos fisiológicos, e pela dificuldade de obtenção de dados relacionados ao clima e ao solo.

Nesse contexto, a utilização de modelos empíricos torna-se uma ferramenta viável, capaz de representar, de forma simplificada, as relações entre a resposta fisiológica das plantas e as variáveis ambientais, durante os diferentes estádios fenológicos de uma cultura (Fonseca et al., 2007; Tonato et al., 2010). A decisão sobre as variáveis a serem utilizadas baseia-se em relações empíricas, obtidas por meio de conhecimento experimental.

O objetivo deste estudo foi gerar modelos empíricos de regressão linear, para a predição da taxa de acúmulo de matéria seca de capim-marandu-Urochloa brizantha (Hochst. ex A. Rich.) R.D. Webster cv. Marandu - em função de variáveis agrometeorológicas.

\section{Material e métodos}

Para gerar os modelos empíricos, foram utilizados dados da taxa média de acúmulo de matéria seca(TAMS) de capim-marandu, cultivado em condições de sequeiro, relativos a 29 períodos de crescimento entre 1998 e 2002, na Embrapa Pecuária Sudeste, São Carlos, SP, a $21^{\circ} 57^{\prime}$ S, $47^{\circ} 51^{\prime} \mathrm{W}$, e à altitude de $860 \mathrm{~m}$. O clima, segundo a classificação de Köppen é do tipo Cwa, tropical de altitude, e o solo é classificado como Latossolo Vermelho-Amarelo. Cada período de crescimento representou média de dez amostragens de forragem, em quadrados de $0,5 \times 0,5 \mathrm{~m}$. As amostras foram secas em estufa de circulação de ar a $65^{\circ} \mathrm{C}$, até atingirem peso constante. A TAMS foi calculada pela divisão da massa de forragem coletada acima da altura de resíduo $(25 \mathrm{~cm})$, pelo número de dias de descanso, que foi, em média, de 35 dias no verão (primavera/verão) e de 56 dias no inverno (outono/inverno).
As variáveis agrometeorológicas avaliadas foram temperatura mínima $\left(\mathrm{T}_{\text {mín }}\right)$, máxima $\left(\mathrm{T}_{\text {máx }}\right)$ e média $\left(\mathrm{T}_{\text {méd }}\right)$ radiação global (Rg), graus-dia (GD), evapotranspiração real (ETR) e potencial (ETP), unidades fototérmicas (UF) e o índice climático de crescimento (ICC). A essas variáveis, com exceção da ETR, foi adicionado um fator de penalização de disponibilidade hídrica, dado pela relação ETR/ETP. O balanço hídrico climatológico (ETR e ETP) foi calculado com base no método de Thornthwaite \& Mather (1955), em escala quinquidial.

As variáveis agrometeorológicas $\mathrm{T}_{\text {mín }}, \mathrm{T}_{\text {máx }}, \mathrm{T}_{\text {méd }} \mathrm{e}$ $\mathrm{Rg}$ foram obtidas por meio de estação metereológica automática de coleta de dados, situada na Embrapa Pecuária Sudeste, distante $800 \mathrm{~m}$ do experimento. Para a análise da regressão, foram utilizados os valores médios das variáveis agrometeorológicas em cada período de crescimento.

A temperatura base inferior (Tbi, $17,2^{\circ} \mathrm{C}$ ) foi obtida pela relação dos dados de TAMS (utilizados na geração dos modelos) com os dados de temperatura média do ar, em cada período de crescimento: TAMS $=a+b \times T_{\text {méd, }}$ em que a e b são os coeficientes linear e angular da regressão. Quando a TAMS for igual a zero, a $\mathrm{T}_{\text {méd }}$ será igual a Tbi $(\mathrm{Tbi}=-\mathrm{a} / \mathrm{b})$.

O GD foi calculado pelas equações: $G D=T_{\text {méd }}-T b i$, quando $\mathrm{T}_{\text {méd }}>\mathrm{Tbi}$; GD $=\left(\mathrm{T}_{\text {máx }}-\mathrm{T}_{\text {méd }}\right)^{2} / 2\left(\mathrm{~T}_{\text {máx }}-\mathrm{T}_{\text {mín }}\right)$, quando $\mathrm{T}_{\text {méd }}=\mathrm{Tbi}$; e GD $=\left(\mathrm{T}_{\text {máx }}-\mathrm{Tbi}\right)^{2} / 2\left(\mathrm{~T}_{\text {máx }}-\mathrm{T}_{\text {mín }}\right)$, quando $\mathrm{T}_{\text {méd }}<\mathrm{Tbi}$.

As UF foram calculadas pela seguinte equação: $\mathrm{UF}=(\mathrm{n} / 2 \times \mathrm{GD})^{\mathrm{Nf} / \mathrm{Ni}+1} /(\mathrm{Nf} / \mathrm{Ni}+1)$, em que GD são os graus-dia e $\mathrm{Nf}$ e $\mathrm{Ni}$ representam o fotoperíodo inicial e final, conforme $\mathrm{N}=24 / \pi \times \cos ^{-1}[\cos (109 / 106 \times \pi)$ $\operatorname{sen}(\lambda) \times \operatorname{sen}(\phi)] /[\cos (\lambda) \times \cos (\phi)]$, em que $\lambda$ é a latitude e $\phi$ é a declinação solar, dada por $\phi=23,5 \pi / 180 \times$ $\cos [2 \pi / 365 \times($ DOY - 173)], em que DOY é o dia juliano.

O ICC foi calculado pela equação $\mathrm{ICC}=\mathrm{IL} \times$ IT $\times$ IH, em que: IL é o índice de luz dado por $\mathrm{IL}=1-\exp [-35(\mathrm{Rg} / 750)] ;$ IT é o índice térmico de gramíneas tropicais, a partir de tabelas apresentadas por Mota et al. (1981), que leva em consideração as temperaturas acima ou abaixo da temperatura ótima; e IH é o índice hídrico, representado pela relação ETR/ETP.

Os dados relacionados à TAMS e às variáveis agrometeorológicas foram submetidos a análises exploratórias preliminares, para eliminar dados 
discrepantes ("outliers") e obedecer às premissas básicas da análise de variância (linearidade, homoscedasticidade e normalidade dos erros). Na variável $\mathrm{T}_{\text {máx }}$, foi utilizada a técnica dos mínimos quadrados ponderados no ajuste dos parâmetros, em virtude de problemas com as premissas estatísticas.

Após as análises preliminares, foram realizadas análises de regressões lineares simples $(y=a+b x)$ e múltiplas, entre as variáveis agrometeorológicas (variáveis independentes) e a TAMS (variável dependente). Para a regressão linear simples, foi utilizado o Proc Reg do SAS (Littel et al., 2006) e, para a regressão linear múltipla, foi utilizado o Proc Stepwise (passo a passo), que indicou as variáveis independentes com melhor ajuste. A seleção dos modelos baseou-se no coeficiente de determinação $\left(\mathrm{R}^{2}\right)$, na raiz do quadrado médio do resíduo (RQMR) e no critério de informação de Akaike (CIA). O CIA foi obtido com o auxílio do Proc Mixed do SAS, pela expressão CIA $=2 \mathrm{~K}-2 \ln (\mathrm{L})$, em que $\mathrm{K}$ é o número de parâmetros, e L é o valor de máxima verossimilhança da função.

Para a validação dos modelos, foram utilizados 35 períodos distintos de produção de forragem, entre 2005 e 2006, em Piracicaba, SP, conforme Lara (2007) e Bertolote (2009); e entre 2009 e 2010 conforme Cruz (2010) em São Carlos, SP. Todos os experimentos utilizados na validação foram conduzidos sem restrição hídrica ou nutricional.

O desempenho dos modelos escolhidos foi avaliado pelo índice d de Willmott, pelo índice de confiança, c, conforme Camargo \& Sentelhas (1997), e pelo quadrado médio do erro de predição (QMEP). O índice c permite uma interpretação objetiva para os testes de acurácia, representado pelo índice $\mathrm{d}$, e de precisão, representada pelo coeficiente de correlação, que varia de ótimo $(>0,85)$ a muito bom $(0,76-0,85)$, bom $(0,66-$ $0,75)$, mediano $(0,61-0,65)$, sofrível $(0,51-0,60)$, mau $(0,41-0,50)$ e péssimo $(\leq 0,40)$, a depender do resultado da razão entre eles.

$\mathrm{O}$ índice $\mathrm{d}$ foi obtido pela equação,

$$
\mathrm{d}=1-\Sigma(\mathrm{Pi}-\mathrm{Oi})^{2} / \Sigma(|\mathrm{Pi}-\mathrm{Oi}|+|\mathrm{Oi}-\mathrm{O}|)^{2},
$$

em que: Pi é o valor predito, Oi é o observado; e O é a média dos valores observados.

$\mathrm{O}$ índice $\mathrm{c}$ foi obtido pela razão entre o índice $\mathrm{d}$ e o coeficiente de correlação $(r): c=r \times d$.
O QMEP foi calculado pela equação,

$$
\mathrm{QMEP}=\sum_{\mathrm{i}=1}^{\mathrm{n}}\left[\mathrm{Oi}-\mathrm{f}\left(\mathrm{P}_{1} \ldots . \mathrm{P}_{\mathrm{p}}\right) \mathrm{i}\right]^{2} / \mathrm{n},
$$

em que: Oi são os valores observados; P são os valores preditos pelo modelo; e n é o número de observações.

\section{Resultados e Discussão}

As análises de regressão lineares univariada e multivariada, entre a TAMS de Urochloa brizantha cv. Marandu e as ocorrências meteorológicas nos períodos de crescimento, estão apresentadas na Tabela 1.

A UF apresentou o menor desempenho entre as variáveis avaliadas, mesmo quando introduzido o fator hídrico (UFcorr). Um dos motivos para o modelo usando UF ter apresentado baixo $\mathrm{R}^{2}(0,17)$ pode ter sido o uso de longos períodos de descanso (em média 56 dias) na geração do modelo, principalmente no inverno, o que teria contribuído para superestimar as UF. Na literatura, encontram-se diferentes resultados de correlações entre TAMS e UF. Lara (2007) encontrou baixa correlação entre a TAMS e as UF acumuladas em cultivares de Urochloa spp.; no capim-marandu, a correlação observada pelos autores não foi significativa. Tonato (2003) observou alta correlação entre as UF e a TAMS em Cynodon spp., com 28 e 42 dias de descanso, independentemente da estação do ano.

Os melhores resultados foram observados para a regressão linear multivariada (MRE), com emprego das variáveis $T_{\text {mín }}, R g$ e ETR, que apresentaram os melhores valores de $\mathrm{R}^{2}$, RQMR e CIA (Tabela 1).

Nas equações de regressão linear univariada, destacaram-se as variáveis preditoras GDcorr e $\mathrm{T}_{\text {míncorr }}$ - em que corr significa a correção pela relação ETR/ ETP - e ICC. Essas variáveis apresentaram os maiores valores de $\mathrm{R}^{2}$ e os menores de RQMR e CIA.

De maneira geral, todas as variáveis agrometeorológicas avaliadas melhoraram seus resultados, quando o fator de disponibilidade hídrica foi inserido, o que mostra a importância da água como determinante na produção de forragem. A variável que compôs o balanço hídrico foi a razão ETR/ETP, capaz de medir o suprimento hídrico para a planta em relação a sua necessidade.

No Brasil há bons resultados que relacionam o ICC como variável climática preditora de produção de forragem (Mota et al.,1981; Santos et al., 2008). Uma das dificuldades encontradas ao se trabalhar com o ICC 
é a obtenção de dados de radiação global, deficiente em muitas estações meteorológicas no Brasil.

As variáveis GDcorr e $T_{\text {míncorr }}$ também apresentaram altas correlações com a TAMS, apesar de envolverem apenas dois fatores agrometeorológicos (temperatura e deficit hídrico). Estudos mostram boas correlações entre a temperatura e a produção de matéria seca no capim-marandu (Rodrigues, 2004; Lara, 2007; Tonato et al., 2010). Rolim (1994) observou que o efeito da temperatura é mais importante na produção de forragem do que o do fotoperíodo. Costa et al. (2005) observaram que, para a região Centro-Oeste brasileira, onde se encontra a maior área plantada com capim-marandu, os principais efeitos do clima na produção são decorrentes das baixas temperaturas e precipitações no período de inverno.

Uma das limitações para a utilização das variáveis GDcorr e ICC está relacionada à determinação das temperaturas basais. Cooper \& Tainton (1968) generalizam entre 10 e $15^{\circ} \mathrm{C}$ as temperaturas bases inferiores (Tbi) para forrageiras tropicais. Entretanto, em estudos com capim-marandu, Rodrigues (2004) e Lara (2007) encontraram valores de Tbi de 18,6 e $16,3^{\circ} \mathrm{C}$, respectivamente. A média desses valores $\left(17,5^{\circ} \mathrm{C}\right)$ está próxima à dos observados e utilizados na geração dos modelos preditores $\left(17,2^{\circ} \mathrm{C}\right)$. A preferência pela variável climática de temperatura tem ocorrido principalmente em razão de sua boa capacidade de estimação de produção e pela facilidade de sua obtenção, em comparação a outras variáveis agrometeorológicas, como por exemplo, a radiação.

Em regiões tropicais e subtropicais, entre as latitudes $30^{\circ} \mathrm{N}$ e $\mathrm{S}$, a temperatura e a deficiência hídrica e sua associação, são os principais fatores limitantes da produção de forragem, diferentemente do que ocorre em regiões temperadas, onde a luz passa a ter grande relevância, seguida da temperatura e da oferta hídrica (McDowell, 1972).

A comparação dos valores preditos pelos modelos agrometeorológicos univariados, para GDcorr, $\mathrm{T}_{\text {míncorr, }}$ e ICC, e multivariados, para $\mathrm{T}_{\text {mín, }}$ Rg e ETR, com os dados observados em experimentos nas cidades de São Carlos e Piracicaba, SP, pode ser observada na Tabela 2. Os modelos apresentaram resultados distintos na capacidade de predição da TAMS. Em Piracicaba, apresentaram índice c de 0,37 a 0,64 , enquanto em São Carlos, esse índice variou de 0,45 a 0,71 . Altas correlações ( $r$ ) foram observadas entre as variáveis agrometeorológicas preditoras e a TAMS ( $\mathrm{r}$ acima de 0,75), com exceção do modelo multivariado em São Carlos. O modelo que apresentou os melhores índices na estimativa de TAMS, em São Carlos, foi o que relacionou os valores de GDcorre, em Piracicaba, o MRE multivariado com três variáveis agrometeorológicas. A Figura 1 mostra as TAMS preditas e observadas, nos diferentes períodos de crescimento, em São Carlos e

Tabela 1. Equações de regressão entre taxa média de acúmulo de matéria seca (TAMS) e as variáveis agrometeorológicas.

\begin{tabular}{|c|c|c|c|c|c|}
\hline Modelo $^{(1)}$ & Equação & $\mathrm{R}^{2}$ & RQMR & CIA & $\mathrm{p}$ \\
\hline \multicolumn{6}{|l|}{ Univariado } \\
\hline Tmáx & TAMS $=14,30 \mathrm{~T}_{\text {máx }}-330,68$ & 0,44 & 26,36 & 263,0 & $<0,0001$ \\
\hline Tmín & TAMS $=11,93 \mathrm{~T}_{\min }-134,95$ & 0,73 & 18,45 & 244,5 & $<0,0001$ \\
\hline Tméd & $\mathrm{TAMS}=14,08 \mathrm{~T}_{\text {méd }}-242,47$ & 0,64 & 21,24 & 251,7 & $<0,0001$ \\
\hline $\mathrm{Rg}$ & $\mathrm{TAMS}=6,21 \mathrm{Rg}-52,92$ & 0,36 & 28,37 & 268,4 & 0,0006 \\
\hline GD & TAMS $=13,86 \mathrm{GD}-6,92$ & 0,64 & 21,24 & 251,7 & $<0,0001$ \\
\hline ETR & $\mathrm{TAMS}=25,46 \mathrm{ETR}-2,69$ & 0,73 & 18,48 & 243,1 & $<0,0001$ \\
\hline ICC & $\mathrm{TAMS}=233,54 \mathrm{ICC}+7,99$ & 0,74 & 17,85 & 236,9 & $<0,0001$ \\
\hline UF & $\mathrm{TAMS}=0,003 \mathrm{UF}+40,42$ & 0,17 & 32,17 & 290,1 & 0,025 \\
\hline Tmáxcorr & $\mathrm{TAMS}=4,11 \mathrm{~T}_{\text {maxcorr }}+29,61$ & 0,69 & 19,77 & 250,4 & $<0,0001$ \\
\hline Tmíncorr & $\mathrm{TAMS}=5,78 \mathrm{~T}_{\text {mincorr }}-17,24$ & 0,75 & 17,82 & 244,1 & $<0,0001$ \\
\hline Tmédcorr & $\mathrm{TAMS}=4,83 \mathrm{~T}_{\text {médcorr }}-24,83$ & 0,71 & 18,89 & 247,6 & $<0,0001$ \\
\hline Rgcorr & $\mathrm{TAMS}=4,44 \mathrm{Rg}_{\text {corr }}-6,36$ & 0,59 & 22,5 & 257,0 & $<0,0001$ \\
\hline GDcorr & $\mathrm{TAMS}=12,9 \mathrm{GD}_{\text {corr }}+6,52$ & 0,75 & 17,84 & 242,6 & $<0,0001$ \\
\hline UFcorr & $\mathrm{TAMS}=0,004 \mathrm{UF}_{\text {corr }}+33,45$ & 0,35 & 28,37 & 281,1 & 0,0006 \\
\hline Multivariado & $\mathrm{TAMS}=8,3 \mathrm{~T}_{\min ^{\prime}}-5,2 \mathrm{Rg}+21,9 \mathrm{ETR}-36,3$ & 0,84 & 14,72 & 222,5 & $<0,0001$ \\
\hline
\end{tabular}

${ }^{(1)} \mathrm{T}_{\operatorname{maxx}}, \mathrm{T}_{\text {min }}, \mathrm{T}_{\text {méd }}$ são, respectivamente, as temperaturas máxima, mínima e média em ${ }^{\circ} \mathrm{C}$; $\mathrm{Rg}$ é a radiação em $\mathrm{MJ} \mathrm{m}^{-2} ; \mathrm{GD}$ é grau-dia $\left({ }^{\circ} \mathrm{C}\right)$; $\mathrm{ETR}$ é a evapotranspiração real em mm; ICC é o índice climático de crescimento; UF são as unidades fototérmicas; MRE é a regressão linear múltipla; corr é o fator de correção que representa a relação ETR/ETP. R², coeficiente de determinação; RQMR, raiz do quadrado médio do resíduo; CIA, critério de informação de Akaike; e p, significância estatística. 
Tabela 2. Valores de índice de Willmott (d), coeficiente de determinação $\left(\mathrm{R}^{2}\right)$, coeficiente de correlação $(\mathrm{r})$, quadrado médio do erro de predição (QMEP) e índice de confiança (c) dos modelos agroclimáticos de predição da taxa média de acúmulo de matéria seca (TAMS), utilizados para dados obtidos em São Carlos e Piracicaba, SP.

\begin{tabular}{|c|c|c|c|c|c|c|}
\hline Modelo $^{(1)}$ & Índice $\mathrm{d}$ & $\mathrm{R}^{2}$ & $\mathrm{r}$ & QMEP & Índice $\mathrm{c}$ & Desempenho \\
\hline & \multicolumn{6}{|c|}{ São Carlos } \\
\hline \multicolumn{7}{|l|}{ Univariado } \\
\hline $\mathrm{GD}_{\text {corr }}$ & 0,88 & 0,66 & 0,81 & 352 & 0,71 & Bom \\
\hline ICC & 0,85 & 0,57 & 0,76 & 361 & 0,65 & Mediano \\
\hline $\mathrm{T}_{\text {mincorr }}$ & 0,67 & 0,76 & 0,87 & 671 & 0,58 & Sofrível \\
\hline Multivariado & 0,75 & 0,35 & 0,59 & 879 & 0,45 & Mau \\
\hline & \multicolumn{6}{|c|}{ Piracicaba } \\
\hline \multicolumn{7}{|l|}{ Univariado } \\
\hline $\mathrm{GD}_{\text {corr }}$ & 0,56 & 0,59 & 0,77 & 1.761 & 0,43 & Mau \\
\hline ICC & 0,48 & 0,60 & 0,77 & 2.029 & 0,37 & Péssimo \\
\hline $\mathrm{T}_{\text {mincorr }}$ & 0,48 & 0,58 & 0,76 & 1.684 & 0,37 & Péssimo \\
\hline Multivariado & 0,71 & 0,82 & 0,90 & 1.335 & 0,64 & Mediano \\
\hline
\end{tabular}

${ }^{(1)} \mathrm{GD}_{\text {corr, }}$ graus-dia corrigido pela relação ETR/ETP; ICC, índice climático de crescimento; $\mathrm{T}_{\text {mincorr, }}$ temperatura mínima corrigida pela relação ETR/ETP.
Piracicaba. Em Piracicaba, houve superestimativa da TAMS predita pelos modelos. Isso está relacionado ao fato de a média das TAMS observadas em Piracicaba, para o inverno e verão, serem de 14,4 e $49,7 \mathrm{~kg} \mathrm{ha}^{-1}$ por dia, respectivamente, contra 42,6 e $81,9 \mathrm{~kg} \mathrm{ha}^{-1}$ por dia em São Carlos.

As limitações observadas na estimativa da TAMS a partir das variáveis agrometeorológicas, em Piracicaba, estão possivelmente ligadas às características intrínsecas do conjunto de dados utilizados no teste. Uma delas pode estar relacionada ao fato de os dados terem sido gerados em um único experimento, manejado de forma distinta dos utilizados na geração dos modelos, principalmente em relação à altura e frequência de corte. A altura do resíduo, nos dados utilizados para gerar os modelos, foi de $25 \mathrm{~cm}$, com frequências de cortes em média de 56 dias no inverno, diferentemente dos $15 \mathrm{~cm}$ de resíduo e 42 dias de frequência no inverno avaliados em Piracicaba.
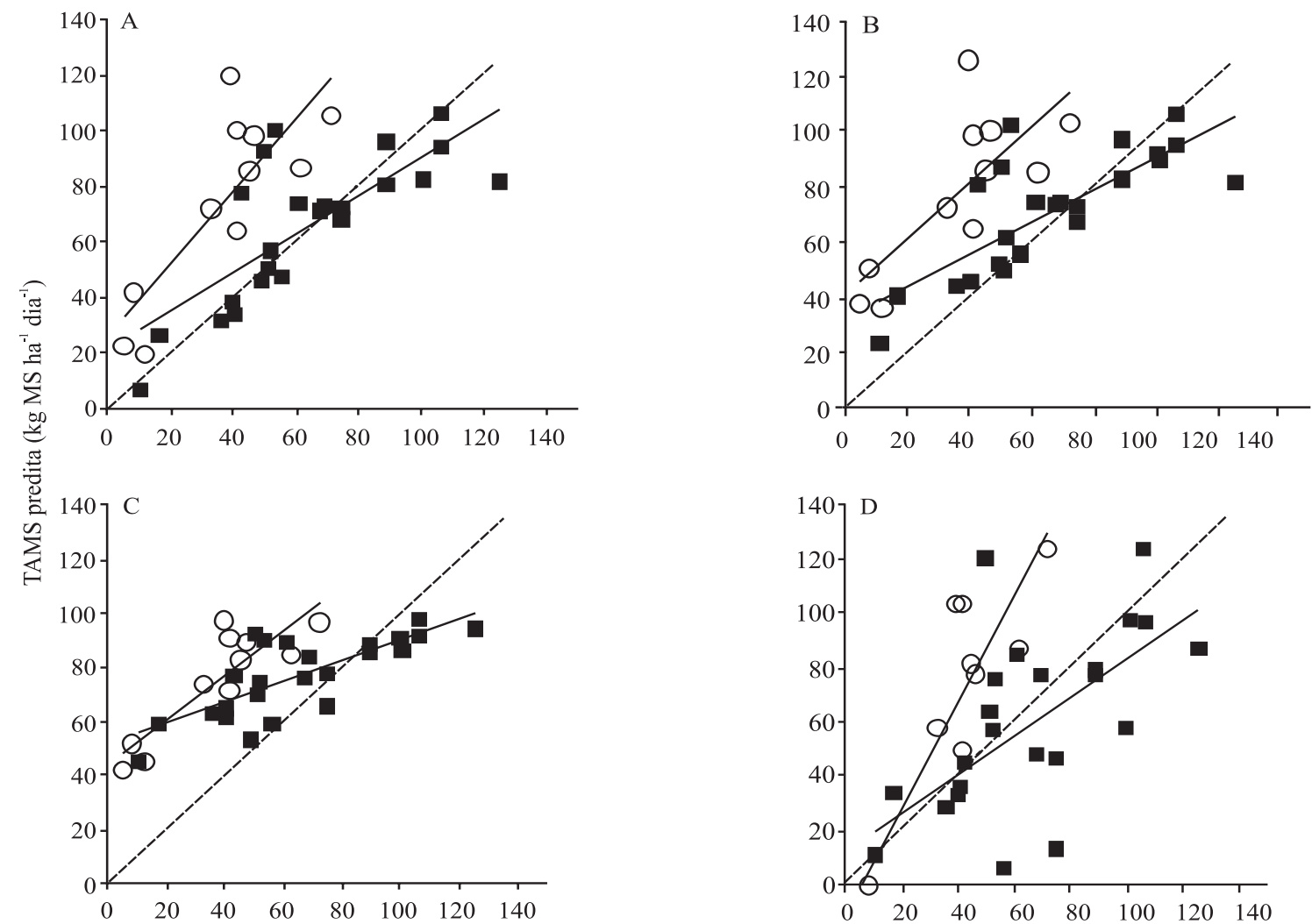

TAMS observada $\left(\mathrm{kg} \mathrm{MS} \mathrm{ha}^{-1} \mathrm{dia}^{-1}\right)$

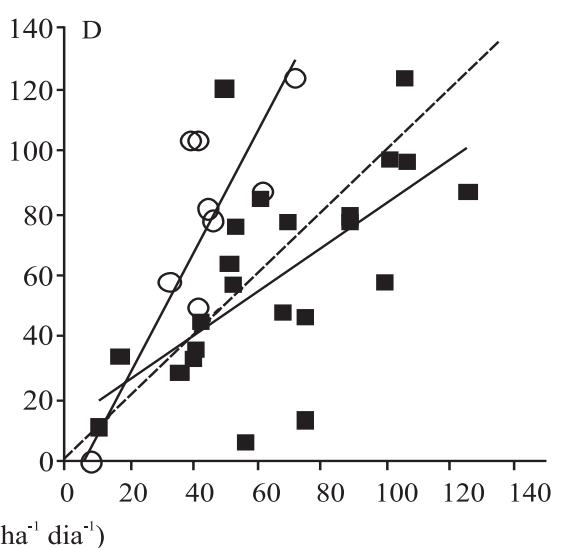

Figura 1. Relação entre a taxa média de acúmulo de matéria seca (TAMS) predita e observada, com uso do modelo univariado e emprego das variáveis climáticas: graus-dia corrigido (A); índice climático de crescimento (B); temperatura mínima corrigida $(\mathrm{C})$; ou do modelo multivariado com emprego das variáveis: temperatura mínima, radiação e evapotranspiração real (D), em 35 períodos de crescimento em São Carlos (ם) e Piracicaba (o), SP. 
Apesar da baixa acurácia observada nas estimativas de TAMS, em Piracicaba, altos valores de correlação foram observados entre as variáveis agrometeorológicas e a estimativa de produção.

Uma possível solução para melhorar a acurácia dos dados é apresentada por Tonato et al. (2010), que assumiram o valor de Tbi como uma constante. No presente trabalho, os autores realizaram uma calibração para $U$. decumbens cv. Basilisk com dados de produção de forragem em uma propriedade rural, tendo elevado, assim, a acurácia das estimativas de produção. A calibração foi realizada pelo ajuste do coeficiente angular do modelo, com base no valor inicial da soma de quadrados dos desvios, o que minimizou a soma de quadrado dos resíduos. Fica evidente a importância da calibração dos modelos com dados locais para promover os ajustes dos parâmetros (Tonato et al., 2010).

Uma das limitações associadas ao conjunto de dados utilizado na geração dos modelos é a pequena abrangência geográfica, já que os modelos foram gerados apenas com dados referentes a São Carlos. Modelos empíricos com variáveis agrometeorológicas fornecem boas estimativas de produção, mas são restritos às condições do meio e à cultivar com os quais foram gerados (Goudriaan \& Laar, 1994).

\section{Conclusões}

1. As regressões univariada e multivariada mostraram boa capacidade de predição para as taxas médias de acúmulo diário de matéria seca (TAMS) de Urochloa brizantha cv. Marandu.

2. As predições mais precisas de TAMS são obtidas com o uso de regressão multivariada, com emprego das variáveis temperatura mínima, radiação global e evapotranspiração real.

3. A correção das variáveis agrometeorológicas pela relação entre evapotranspiração real e potencial (ETR/ ETP), em geral, melhora a predição da TAMS.

4. Para a regressão univariada, destacam-se as variáveis graus-dia e temperatura mínima, corrigidas pela relação ETR/ETP, bem como o índice climático de crescimento.

\section{Referências}

BERTOLOTE, L.E.M. Sobressemeadura de forrageiras de clima temperado em pastagens tropicais. 2009. 83p. Dissertação (Mestrado) - Faculdade de Medicina Veterinária e Zootecnia, Botucatu.
BOOTE, K.J.; MINGUEZ, M.I.; SAU, F. Adapting the CROPGRO legume model to simulate growth of faba bean. Agronomy Journal, v.94, p.743-756, 2002.

CAMARGO, A.P. de; SENTELHAS, P.C. Avaliação do desempenho de diferentes métodos de estimativa da evapotranspiração potencial no Estado de São Paulo, Brasil. Revista Brasileira de Agrometeorologia, v.5, p.89-97, 1997.

COOPER, J.P.; TAINTON, N.M. Light and temperature requirements for growth of tropical and temperate grasses. Herbage Abstracts, v.38, p.167-176, 1968.

CORSON, M.S.; ROTZ, C.A.; SKINNER, R.H. Evaluating warmseason grass production in temperate-region pastures: a simulation approach. Agricultural Systems, v.93, p.252-268, 2007.

COSTA, K.A. de P.; ROSA, B.; OLIVEIRA, I.P. de; CUSTÓDIO, D.P.; SILVA, D.C. e. Efeito da estacionalidade de produção de matéria seca e composição bromatológica da Brachiaria brizantha cv. Marandu. Ciência Animal Brasileira, v.6, p.187-193, 2005.

CRUZ, P.G. da. Produção de forragem em Brachiaria brizantha: adaptação, geração e avaliação de modelos empíricos e mecanicistas para a estimativa do acúmulo de forragem. 2010. 102p. Tese (Doutorado) - Escola Superior de Agricultura Luiz de Queiroz, Piracicaba.

FONSECA, E.L. da; PONZONI, F.G.; FORMAGGIO, A.R. Modelo agrometeorológico-espectral para estimativa da disponibilidade de forragem no bioma "Campos Sulinos". Revista Brasileira de Agrometeorologia, v.15, p.241-249, 2007.

GOUDRIAAN, J.; LAAR, H.H. van. Modeling potential crop growth processes: textbook with exercises. Dordrecht: Kluwer Academic Publishers, 1994. 238p.

LARA, M.A.S. Respostas morfofisiológicas de cinco cultivares de Brachiaria spp. às variações estacionais da temperatura do ar e do fotoperíodo. 2007. 91p. Dissertação (Mestrado) - Escola Superior de Agricultura Luiz de Queiroz, Piracicaba.

LITTELL, R.C.; MILLIKEN, G.A.; STROUP, W.W.; WOLFINGER, R.D.; SCHABENBERGER, O. Sas for mixed models. $2^{\text {nd }}$ ed. Cary: SAS Institute, 2006. 813p.

MCDOWELL, R.G. Improvement of livestock production in warm climates. San Francisco: W. H. Freeman and Company, 1972. 771p.

MOTA, F.S. da; BERNY, Z.B.; MOTA, J.F.A.S. da. Índice climático de crescimento de pastagens naturais no Rio Grande do Sul. Pesquisa Agropecuária Brasileira, v.16, p.453-472, 1981.

PEDREIRA, B.C.; PEDREIRA, C.G.S.; BOOTE, K.J.; LARA, M.A.S.; ALDERMAN, P.D. Adapting the CROPGRO perennial forage model to predict growth of Brachiaria brizantha. Field Crops Research, v.120, p.370-379, 2011.

RODRIGUES, D.C. Produção de forragem de cultivares de Brachiaria brizantha (Hochst. ex. A. Rich.) Stapf. e modelagem de respostas produtivas em função de variáveis climáticas. 2004. 94p. Dissertação (Mestrado) - Escola Superior de Agricultura Luiz de Queiroz, Piracicaba. 
ROLIM, F.A. Estacionalidade de produção de forrageiras. In: PEIXOTO, A.M.; MOURA, J.C. de; FARIA, V.P. de (Ed.). Pastagens: fundamentos da exploração racional. 2.ed. Piracicaba: FEALQ, 1994. p.533-565.

RYMPH, S.J.; BOOTE, K.J.; IRMAK, A.; MISLEVY, P.; EVERS, G.W. Adapting the CROPGRO model to predict growth and composition of tropical grasses: developing physiological parameters. Soil and Crop Science Society of Florida Proceedings, v.63, p.37-51, 2004.

SANTOS, F.G. dos; CHAVES, M.A.; SILVA, M.W.R. da; SOARES, R.D.; FRANCO, I.L.; PINHO, B.D. Índice climático de crescimento para os capins Brachiaria brizantha cv. Marandu, Cynodon dactylon cv. Tifton 85 e Panicum maximum cv. Tanzânia e relação com a produção de massa seca. Ciência Animal Brasileira, v.9, p.627-637, 2008.

SOLOMON, S.; QIN, D.; MANNING, M.; MARQUIS, M.; AVERYT, K.B.; TIGNOR, M.; MILLER JUNIOR, H.L.; CHEN, Z. Climate change 2007: the physical science basis: contribution of working group I to the fourth assessment report of the Intergovernmental Panel on Climate Change. New York: Cambridge University Press, Cambridge, 2007. 989p.

THORNTHWAITE, C.W.; MATHER, J.R. The water balance. New Jersey: Drexel Institute of Technology, 1955. 104p.

TONATO, F. Determinação de parâmetros produtivos e qualitativos de Cynodon spp. em função de variáveis climáticas. 2003. 85p. Dissertação (Mestrado) - Escola Superior de Agricultura Luiz de Queiroz, Piracicaba.

TONATO, F.; BARIONI, L.G.; PEDREIRA, C.G.S.; SILVA, O.D.D. da; MALAQUIAS, J.V. Desenvolvimento de modelos preditores de acúmulo de forragem em pastagens tropicais. Pesquisa Agropecuária Brasileira, v.45, p.522-529, 2010.

ZHU, X.G.; LONG, S.P.; ORT, D.R. What is the maximum efficiency with which photosynthesis can convert solar energy into biomass? Current Opinion in Biotechnology, v.19, p.153-159, 2008 .

Recebido em 4 de novembro de 2010 e aprovado em 31 de maio de 2011 